University of Wollongong

Research Online

Australian Institute for Innovative Materials -

Papers

Australian Institute for Innovative Materials

$1-1-2016$

Dynamic magneto-optical imaging of superconducting thin films

Frederick Wells

University of Wollongong, fsw793@uowmail.edu.au

Alexey V. Pan

University of Wollongong, pan@uow.edu.au

Stephen Wilson

University of Wollongong, sw514@uowmail.edu.au

Igor Golovchanskiy

University of Wollongong, ig684@uowmail.edu.au

Sergey Fedoseev

University of Wollongong, sf477@uowmail.edu.au

See next page for additional authors

Follow this and additional works at: https://ro.uow.edu.au/aiimpapers

Part of the Engineering Commons, and the Physical Sciences and Mathematics Commons

Research Online is the open access institutional repository for the University of Wollongong. For further information contact the UOW Library: research-pubs@uow.edu.au 


\title{
Dynamic magneto-optical imaging of superconducting thin films
}

\begin{abstract}
We present a novel method for analysis of superconducting thin films using dynamic magneto-optical imaging, revealing hallmarks of flux penetration with temporal resolution around $1 \mathrm{~ms}$ (in the present work) or better. This method involves investigation of transient field and dynamic current distributions, which are calculated by an inversion procedure on the Biot-Savart Law, which we show to be valid under dynamic conditions. We compare and discuss the flux front penetration speed and evolution of current distribution in high quality YBa2Cu30\$\{\}_\{-Idelta $\} \$$ thin films with that of samples deliberately damaged in such a way as to reduce critical current density without causing macroscopic damage.
\end{abstract}

\section{Keywords}

films, magneto, superconducting, dynamic, thin, imaging, optical

Disciplines

Engineering | Physical Sciences and Mathematics

\section{Publication Details}

Wells, F. S., Pan, A. V., Wilson, S., Golovchanskiy, I. A., Fedoseev, S. A. \& Rozenfeld, A. (2016). Dynamic magneto-optical imaging of superconducting thin films. Superconductor Science and Technology, 29 (3), $1-8$.

\section{Authors}

Frederick Wells, Alexey V. Pan, Stephen Wilson, Igor Golovchanskiy, Sergey Fedoseev, and Anatoly B. Rosenfeld 


\title{
Dynamic Magneto-Optical Imaging of Superconducting Thin Films
}

\author{
Frederick S. Wells ${ }^{a}$, Alexey V. Pan ${ }^{a},{ }^{*}$ Stephen Wilson ${ }^{a}$, \\ Igor A. Golovchanskiy ${ }^{a, b, c}$, Sergey A. Fedoseev ${ }^{a, d}$, and Anatoly Rozenfeld ${ }^{d}$ \\ ${ }^{a}$ Institute for Superconducting and Electronic Materials, \\ University of Wollongong, Northfields Avenue, Wollongong, NSW 2522, Australia, \\ ${ }^{b}$ Laboratory of Topological Quantum Phenomena in Superconducting Systems, \\ Moscow Institute of Physics and Technology, State University, \\ 9 Institutskiy per., Dolgoprudny, Moscow Region, 141700, Russia, \\ ${ }^{c}$ Laboratory of Superconducting Metamaterials, National University of \\ Science and Technology MISIS, 4 Leninsky prosp., Moscow, 119049, Russia, \\ ${ }^{d}$ Center for Medical \& Radiation Physics, University of Wollongong, \\ Northfields Avenue, Wollongong, NSW 2522, Australia
}

\begin{abstract}
We present a novel method for analysis of superconducting thin films using dynamic magnetooptical imaging, revealing hallmarks of flux penetration with temporal resolution around $1 \mathrm{~ms}$ (in the present work) or better. This method involves investigation of transient field and dynamic current distributions, which are calculated by an inversion procedure on the Biot-Savart Law, which we show to be valid under dynamic conditions. We compare and discuss the flux front penetration speed and evolution of current distribution in high quality $\mathrm{YBa}_{2} \mathrm{Cu}_{3} \mathrm{O}_{7-\delta}$ thin films with that of samples deliberately damaged in such a way as to reduce critical current density without causing macroscopic damage.
\end{abstract}

\section{INTRODUCTION}

Magneto-Optical imaging of superconducting samples has proven extremely useful for quality assurance purposes, with the ability to quickly analyse the quality of films[1-4] and tapes[1, 5-8] in a non-contact, nondestructive and simple way. This technique shows visually the position of cracks $[5,8]$, weak links $[4,6,7]$ and other micro- and macroscopic defects in the sample[2, 9], however there may be other current-limiting mechanisms that are not so readily visible at the macroscopic scale nor directly dependent on critical current density $[10,11]$.

Magneto-Optical Imaging (MOI) is generally carried out under static field conditions with a constant applied DC field[2, 12-16]. MOI investigations into vortex dynamics or other "dynamic" effects in superconductors typically deal with AC fields [17, 18], or simply consider the effect of changing the magnetic sweep rate on the final stable state[19].

However, in order to fully understand dynamic vortex effects, one must consider not only steady DC or AC fields; but field switching and ramping, which leads to a rich variety of transient field effects. These effects may lead to qualitatively different and unique behaviour. Such transient effects are often neglected due to difficulty of observation, since field and current dynamics in superconductors occur over such short time-scales[19-21, 25].

Magnetic flux penetration into superconducting films occurs on time-scales much smaller than those measurable using many magnetic imaging techniques such as scanning SQUID microscopy, which may require minutes or hours to create a magnetic profile of a sample with

\footnotetext{
* Corresponding author's email: pan@uow.edu.au
}

resolution approaching the vortex scale[26]. High-speed magneto-optical imaging (MOI) overcomes this limitation of other magnetic microscopy techniques as it does not require any scanning, instead relying on the Faraday effect and using visible optics to examine magnetic fields. Therefore dynamic images can be taken, with temporal resolution limits provided only by the exposure time of the camera used and response speed of indicator films.

High-speed magneto-optical imaging and current calculation has been used to investigate dynamic effects such as the relaxation of current distribution in a YBCO strip after a current pulse[20] (though this was limited by a relatively long exposure time), the response to a high frequency AC transport current[21] and the dynamic processes of a thermal quench[22].

Some groups have made modifications to the MOI technique specifically for fast imaging such as laser scanning[23] and polarimetry by varying the polariser angle[24]. These have been shown to increase sensitivity and versatility of the technique, though ultimately at the cost of reduced image acquisition speed.

In this work, we apply high-speed magneto-optical imaging to the investigation of dynamic effects of the magnetic flux in $\mathrm{YBa}_{2} \mathrm{Cu}_{3} \mathrm{O}_{7-\delta}$ (YBCO) superconducting thin films with a focus on achieving the fastest measurements possible. This is achieved by real-time observation of magnetic flux penetration into both highquality and reduced-quality samples, which have vastly differing current carrying ability. The two-dimensional currents in each sample are then calculated independently at each time-step using an inversion procedure of the Biot-Savart Law [2, 27-30], which we show to be valid under dynamic conditions for our measurements (though they are generally quasi-static equations). 


\section{EXPERIMENTAL METHOD}

\section{A. Sample Preparation}

$\mathrm{YBa}_{2} \mathrm{Cu}_{3} \mathrm{O}_{7-\delta}$ films were deposited by pulsed laser deposition on $5 \times 5 \mathrm{~mm}$ substrates of $\mathrm{SrTiO}_{3}$, by the method described in $[31,32]$.

Critical temperature $\left(\mathrm{T}_{c}\right)$ of the samples was measured using a Quantum Design Magnetic Property Measurement System (MPMS) after deposition, and only those with $T_{c} \simeq 90 \mathrm{~K}$ were selected for patterning. The samples were patterned using laser lithography and etched into $3 \times 3 \mathrm{~mm}$ squares with edges sharper than $1 \mu \mathrm{m}$ using ion beam milling.

Most samples were patterned under optimal conditions, however in this work a number of samples were exposed to the ion beam for three times the average etch duration, and subsequently exposed to acetone for an extended period of time.

After patterning, the critical current $\left(J_{c}\right)$ was calculated from MPMS hysteresis measurements for each sample[33, 34] in order to confirm reduction of sample quality and current-carrying ability in the over-processed samples.

\section{B. High-Speed Magneto-Optical Imaging}

The magneto-optical imaging apparatus for high-speed application consists of a polarising microscope modified to view samples under magnetic fields and at low temperature. A Faraday-active indicator film is placed on top of the sample to visualise field, and a high-speed camera used to capture magneto-optical (MO) video. Fig. 1 gives a diagram of the apparatus.

The polarisation of light incident on the indicator film is rotated by an angle proportional to the z-component of local magnetic field in the film, due to the Faraday effect. The operational part of the indicator film is a Faradayactive Bi-doped yttrium-iron garnet film. Such films have been shown to be sensitive magnetic field detectors with undampened response up to at least $10^{6} \mathrm{~Hz}[35]$, which is fast enough to detect any changes in magnetic field observable with our high-speed camera.

In order to create an optical image of the field in the film (which corresponds to stray field above the sample surface), a polariser is placed in the light path before the indicator film, and a crossed analyser after it.

Magnetic field was applied to superconducting samples using Xantrex XKW 40-75 power supply attached to a solenoid with a magnetic ramping speed of $(8.1 \pm 2.3) \mathrm{T} / \mathrm{s}$. For our measurements, the field is increased rapidly from zero to $0.1 \mathrm{~T}$, reaching $90 \%$ of this value within $(27 \pm 3) \mathrm{ms}$, as measured using an SS495A1 Miniature Ratiometric Linear Hall sensor and Agilent 34420A Micro Ohm meter. Values for ramping speed and stabilisation time are averages after measuring many

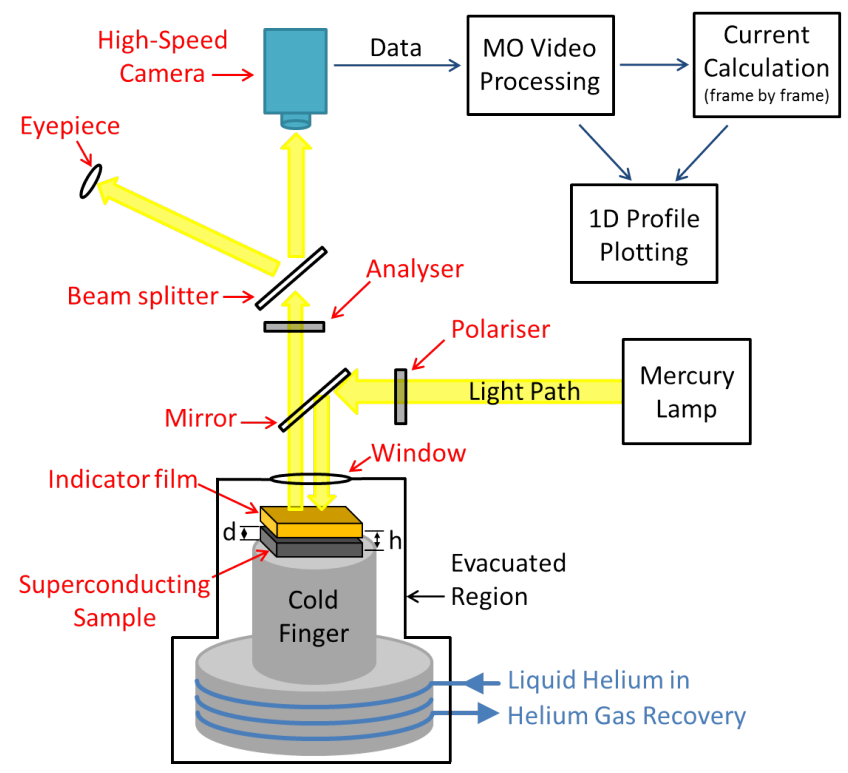

FIG. 1. Diagram of the magneto-optical imaging apparatus for high-speed imaging, showing light path, helium flow and data flow. The geometrical factors $d$ and $h$ are shown, representing the thickness of the sample and height of the indicator film respectively. A solenoid is placed around the cold finger, not shown for simplicity. (colour online)

field-time characteristics. One such curve is shown in fig. 2.

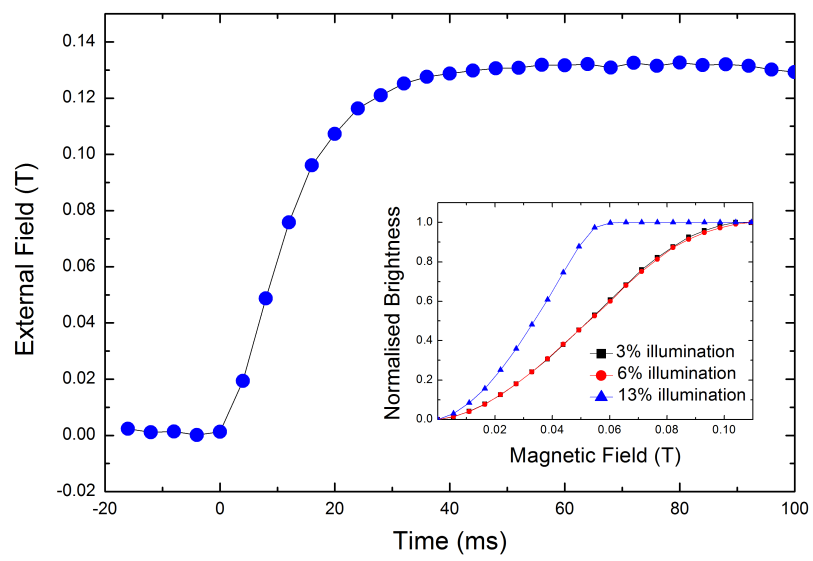

FIG. 2. Typical Hall-sensor characteristic of the solenoid used for external field ramping. The zero time-point is taken from when the field first starts to increase after it is switched on. Inset: Calibration of indicator films using precise field values at various illumination levels.

Sample temperatures of $4 \mathrm{~K}$ and below are maintained using a Janis continuous-flow helium cryostat and a brass cold finger. The cold finger elevates the sample to a position at the centre of the solenoid, where field is most uniform. Cernox temperature sensors at the base and top of the cold finger confirm minimal temperature gradient 
between the sample and the heat exchanger. An inbuilt resistance-heater allows control of the sample temperature and rapid heating above $T_{c}$ in order to re-cool the sample and re-measure penetration.

Samples are illuminated using a Nikon Intensilight CHGFI mercury lamp, and high-resolution images captured through the microscope using a Photron Fastcam SA3 high-speed camera, which is capable of capturing video up to 120,000 frames per second (fps). Each image is interpreted as a direct mapping of the magnetic field around the sample averaged over the exposure time.

An independent calibration is carried out for each video using the brightness of areas of the indicator film far from the superconducting sample as the applied field changes. This calibration data is used to convert the brightness of each pixel to a magnetic field value. A typical calibration curve is shown in the inset of fig. 2. It is fairly linear for most fields applied, but some curvature occurs close to $0.1 \mathrm{~T}$ due to indicator film saturation. Saturation of the camera is also seen for the case of $13 \%$ illumination, but this is strictly avoided in all further measurements.

In this work, magneto-optical videos were captured at the rate of 500 to $2000 \mathrm{fps}$, which is appropriate to the flux penetration speed, allowing investigation of current dynamics and relaxation time. We use an exposure time of 0.5 to $2 \mathrm{~ms}$, and a physical resolution of 150 pixels $/ \mathrm{mm}$. The samples are kept at a constant temperature of $12 \mathrm{~K}$, which was empirically found to be the lowest temperature allowing full penetration in the field range used.

We define full penetration as occurring when the flux fronts from each edge meet, with the only non-flux-filled region being the typical discontinuity lines from corners to centre. The penetration speed into each sample is determined from magneto-optical images by plotting the position of the flux front over time.

To trigger video capture at the moment the applied field is varied, a custom control LabView program was built with a precise timing sequence: first the video recording is triggered, then after a variable delay of a few ms, current to the solenoid is changed in order to increase or decrease the applied field. The camera then continues recording for 0.2 to 10 seconds to also capture the flux creep and/or relaxation effects.

\section{Dynamic Current Calculation}

The current in each frame of the video was calculated independently by inversion of Biot-Savart law. The BiotSavart law gives an expression for field $\mathbf{B}$ at some position $\mathbf{r}$ with respect to a current distribution $\mathbf{J}\left(\mathbf{r}^{\prime}\right)$ :

$$
\mathbf{B}(\mathbf{r})=\frac{\mu_{0}}{4 \pi} \int \frac{\mathbf{J}\left(\mathbf{r}^{\prime}\right) \times\left(\mathbf{r}^{\prime}-\mathbf{r}\right)}{\left|\mathbf{r}^{\prime}-\mathbf{r}\right|^{3}} d^{3} \mathbf{r}^{\prime}
$$

To allow inversion of this equation, we consider sheet currents in the film with minimal variation across the film's small dimension, i.e. $\mathbf{J}(\mathbf{r})=\mathbf{J}_{\mathbf{x}}(\mathbf{x}, \mathbf{y}) \hat{\mathbf{x}}+\mathbf{J}_{\mathbf{y}}(\mathbf{x}, \mathbf{y}) \hat{\mathbf{y}}$ throughout the film's thickness $d[27,28]$. We also consider the film to be locally uncharged, or at least to have local charge varying slowly in time to allow current continuity:

$$
\nabla \cdot \mathbf{J}=0
$$

Fourier transformation of equation (2) gives:

$$
\tilde{J}_{x}\left(k_{x}, k_{y}\right) k_{x}+\tilde{J}_{y}\left(k_{x}, k_{y}\right) k_{y}=0
$$

[28]. Applying equation (1) to the perpendicular magnetic field component $B_{z}$ in the indicator film at height $z=h$ due to sheet currents, and considering equation (3), we acquire by two-dimensional Fourier transformation:

$$
\widetilde{B_{z}}\left(k_{x}, k_{y}, h\right)=i \frac{\mu_{0}}{2} \frac{e^{-h k}}{k_{y}} \sinh \left(\frac{d}{2} k\right) \tilde{J}_{x}\left(k_{x}, k_{y}\right)
$$

$[2,27-30]$. The tilde represents a Fourier-transform of a quantity, $k_{x}$ and $k_{y}$ the frequency components in each direction and $k=\sqrt{k_{x}^{2}+k_{y}^{2}}$.

Equations (4) and (3) are sufficient to calculate the magnitude and direction of current at every point in the film upon inverse Fourier transformation.

In order to better understand the immense amounts of data produced, the time-dependant current profiles in a one-dimensional slice through the sample was considered in favour of the two-dimensional currents over the entire sample.

There are two quasi-static approximations in this determination of currents in the film from perpendicular field measurements in a plane above it, which shall both be now explained and justified for dynamic calculations:

The first is the Biot-Savart law (equation 1), which only holds for quasi-static current distributions, and hence the question may very well be raised as to its validity for calculating currents in our YBCO samples as both field and current change rapidly during flux penetration and depenetration. In vector form this equation can be expressed as

$$
\nabla \times \mathbf{B}=\mu_{0} \mathbf{J}
$$

Which, by comparison to one of Maxwell's equations, makes the assumption that the electric field $E$ varies relatively slowly in time, i.e. $\mathbf{J}>>\epsilon_{0} \frac{\partial \mathbf{E}}{\partial t}[36]$. The electric field in a superconducting sample during charge penetration is proportional to the rate of change of applied magnetic field, and is present only in the penetrated regions[37]. Therefore we calculate the maximum rate of change of electric field from the highest second derivative of the field ramping data (from fig 2).

The maximum value for $\epsilon_{0} \frac{\partial E}{\partial t}$ was found to be $2.55 \times 10^{-5} \mathrm{~A} / \mathrm{m}^{2}$ for our films. Comparing this to the current density in the penetrated region, which exceeds $J=10^{7} \mathrm{~A} / \mathrm{m}^{2}$ within $2 \mathrm{~ms}$ for all samples measured, it is easily seen that this assumption holds. 
The second assumption is that current is continuous in the film, as expressed in equation 2. This assumption relies on currents neither beginning nor ending at any point in the film, meaning the film remains locally uncharged: $\frac{\partial \rho}{\partial t}=0$, where $\rho$ is charge density.

However, investigations into the charge on superconducting films during penetration have shown that square regions near the sample corners do in fact acquire a charge proportional to the rate of external field ramping[37, 38], which may invalidate equation (2) in parts of the film, causing slight inaccuracy in the calculated current in these areas. However, the area around a line through the middle of the film bisecting each edge should always remain charge-free[37]. Therefore we take this line as the position of the one-dimensional slice, as shown in the final current image of figure 4(a), as this lies in the region where calculated currents are most accurate.

\section{RESULTS AND DISCUSSION}

The critical current density $\left(J_{c}\right)$ was measured for each sample using MPMS at $10 \mathrm{~K}$, as shown in figure 3 This initial check shows strong suppression of the critical current density in the over-processed sample by a factor of nearly $10^{5}$ at low fields and complete $J_{c}$ suppression at applied fields $B_{a}>1 \mathrm{~T}$.
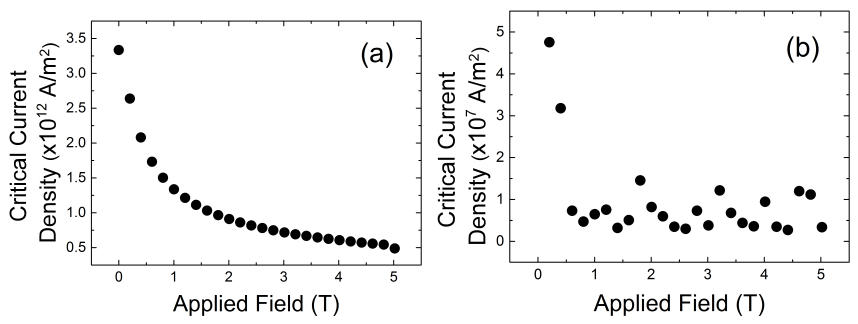

FIG. 3. Critical current density as a function of applied field at $10 \mathrm{~K}$ in (a) the high-quality sample, and (b) the reducedquality sample, where low-field $J_{c}$ is seen to have been reduced by 5 orders of magnitude.

Magneto-optical video showing the dynamic behaviour of field and current in each sample was captured during and immediately after an increase of external field from zero. A selection of frames from typical magneto-optical videos of flux penetration are shown in figure 4 , each frame showing the stray field distribution above the sample at a given point in time. This figure also shows the current in the sample as calculated from the field distribution at each time step. Magneto-optical videos appear visibly similar for both types of films (high quality and degraded quality), therefore frames from only one of the videos are shown in figure 4 . This similarity is important feature, confirming the integrity of superconducting properties in both films. However, further investigation yields important qualitative and quantitative differences in the distribution of field and current in these films.

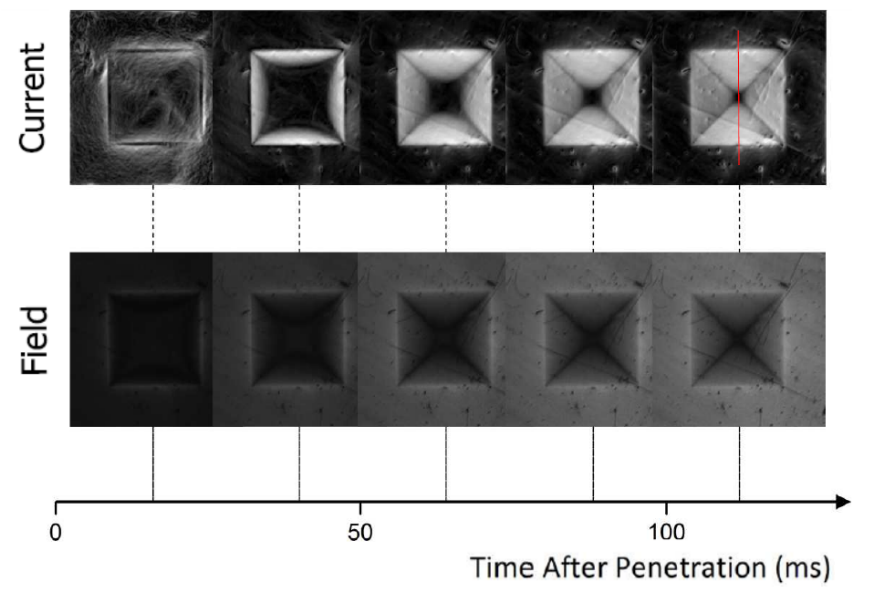

FIG. 4. Timeline of magnetic field and current in a thin film YBCO square at $12 \mathrm{~K}$ during flux penetration. Field images are frames of high-speed magneto-optical video with brightness directly proportional to local magnetic field. Current images are computer-generated with brighter regions representing a higher current magnitude, scaled relative to the maximum current for each time step. The solid (red) line in the top left image shows the position used for one-dimensional current profiles and flux front measurement.

The first quantitative comparison between the samples from the magneto-optical videos involves measuring the penetration speed into each using the motion of the flux fronts, as shown in figure 5.

The magnetic flux front is roughly defined as the line separating flux-filled regions of the sample near its edges and the flux-free region near the centre. During magnetic penetration it moves from the sample edge toward the sample centre. The position of the magnetic flux front was defined to be the furthest point (pixel) from the sample edge with the field above a threshold value $B_{t}$ (chosen to be above the noise level in the flux-free region, but below $10 \%$ of the maximum field), and a field at an adjacent point (pixel) below $B_{t}$, for each time step. This position is plotted against time for each sample in fig 5 , using field values from magneto-optical video along the one-dimensional slice shown in fig 4 . It was noted that both samples had a low sensitivity to changes in the chosen $B_{t}$, since field increases smoothly from zero at the flux front.

From fig 5(a), it can be seen that the flux front penetrates at a relatively constant rate into the high-quality sample for $\sim 50 \mathrm{~ms}$, before slowing gradually. From the gradient of the initial part of these curves, the flux front penetration speeds for this sample were found to be $12.3 \pm 0.2 \mathrm{~mm} / \mathrm{s}$ from one edge and $14.1 \pm 0.1 \mathrm{~mm} / \mathrm{s}$ from the other.

Flux fronts with constant velocity of the order of 100 $\mathrm{m} / \mathrm{s}$ have been theoretically predicted for superconducting samples with field quickly removed[39]. Similar speeds may also be expected for field quickly applied to the sample as in our case, but the speeds we observe ex- 


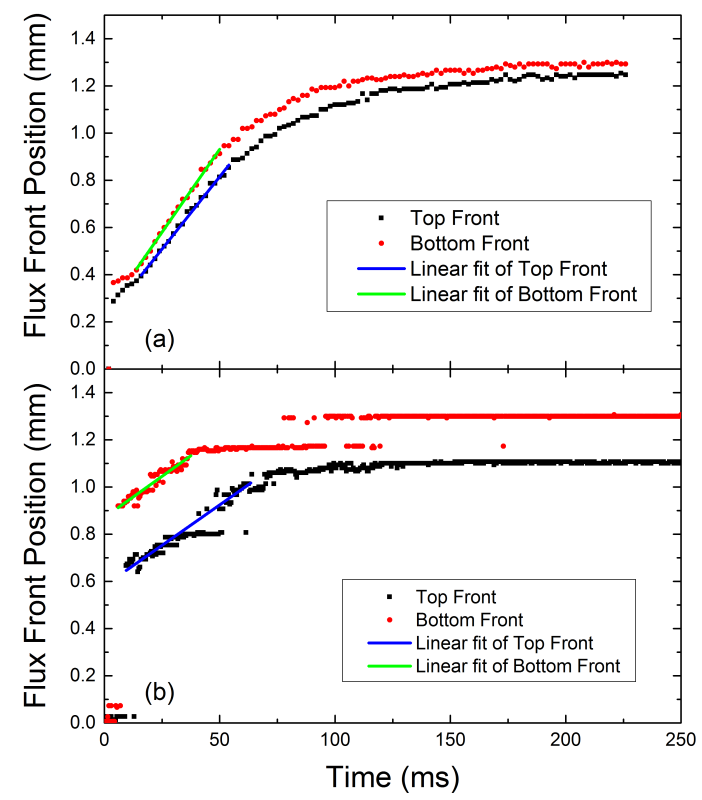

FIG. 5. Position of the flux front over time during penetration in (a) the high-quality sample, and (b) the reduced-quality sample. Flux front position determined from magneto-optical video by finding the furthest point from the film edge with field above a threshold value for each time step. The zero position represents the edge of the measured area. The gradient of these plots gives the penetration speed for each sample.

perimentally are several orders of magnitude lower. This could be due to the dependence of vortex propagation speed on magnetic field ramp rate[40], since the theoretical model considered a very quickly changing applied field[39] while ours ramps at a finite rate.

It should also be noted that the inward speed of vortices during penetration should be fastest somewhere behind the flux front, and is predicted to increase as critical current in the film decreases[40].

It is therefore surprising that flux was seen to penetrate more slowly into the reduced-quality sample in fig 5 (b), with speeds $7.0 \pm 0.3$ and $6.9 \pm 0.2 \mathrm{~mm} / \mathrm{s}$ from each edge. For this sample, the penetration speed does not decrease gradually, but instead appears linear until the final penetrated position, and in this regard more closely reflects the predicted case. Rather than a smooth curve, there is a much sharper corner as the flux front reaches its final position.

Two horizontal lines are also seen in fig $5(\mathrm{~b})$, where the flux front seems to pause before continuing to increase: below $0.8 \mathrm{~mm}$ for the top front, and below $1.2 \mathrm{~mm}$ for the bottom front. These positions are associated with visible defects in the sample.

The unexpected slower flux penetration into the reduced quality film is explained by noting the discontinuity in the plot of fig. 5(b) at approximately $5 \mathrm{~ms}$. This discontinuity represents the time at which the field first penetrates into the sample, and hence the flux front seems to "begin" penetration around $0.6 \mathrm{~mm}$ for the top front, and $0.9 \mathrm{~mm}$ for the bottom front.

However, this position is actually some distance from the sample edge. This "initial" flux front position inside the sample in fact shows a much faster response to the field change, which is too fast to be resolved with the temporal resolution at this frame rate (although it is better than $\sim 1 \mathrm{~ms}$ ). The subsequent (measured) flux speed of $\sim 7 \mathrm{~mm} / \mathrm{s}$, which is lower than in the high quality film is the result of the reduced demagnetising fields, after the initial very fast penetration. This fast penetration is presumably along the damaged domain (grain) boundaries due to the weak interdomain (intergranular) currents, and then slower penetration follows owing to screening currents which lead to lower Lorentz force on the vortices.

Note, we have used the term "interdomain" along with the more conventional "intergranular". This is because it has been established $[1,34,41]$ that our high quality PLD films do not have grains but rather low-angle misoriented domains separated by domain walls consisting of edgedislocations providing strong vortex pinning and critical current, rather than grains connected by weak-links [1].

In order to further quantify the calculated current data, the time-variation of current density in a onedimensional slice was plotted for each film, as shown in figure 6 . One can clearly see that there is an immense change in the behaviour of current density during field penetration in the reduced-quality sample.

Figure 6(a) shows the expected time-evolution of current in a one-dimensional slice through a square YBCO film: A small peak forms at each sample edge within $2 \mathrm{~ms}$ of penetration, which then increases in magnitude without significant change in position until about $20 \mathrm{~ms}$. As this current-peak increases, a long current-tail extends toward the sample centre. By comparison with figure 2, this corresponds to the period of fastest flux ramping. After this, as the external field stabilises, the current peak is seen to move toward the centre of the sample while retaining a constant magnitude. A current plateau forms between this peak and the sample edge, in the penetrated region, with a downward gradient toward the sample edge, which decreases over time. The peak appears to follow the flux front as it penetrates further into the sample, with current decreasing to zero at the centre (position $=1.5 \mathrm{~mm}$ ) at all times.

After about $200 \mathrm{~ms}$, the shape of the current profile in the high-quality sample settles to a distribution resembling that predicted by the Kim-model[13-16], being zero at the sample centre and with a peak at the flux front. Current distribution is quite symmetric about the centre of the sample at all times.

The evolution of the current profile in the reducedquality sample is quite different, as seen in figure 6(b). The peak in current forms at the sample edge as expected, and grows in magnitude during field ramping, though the shape of the current distribution has already deviated after $10 \mathrm{~ms}$, with no tail extending toward the centre (because the flux front has already advanced up 


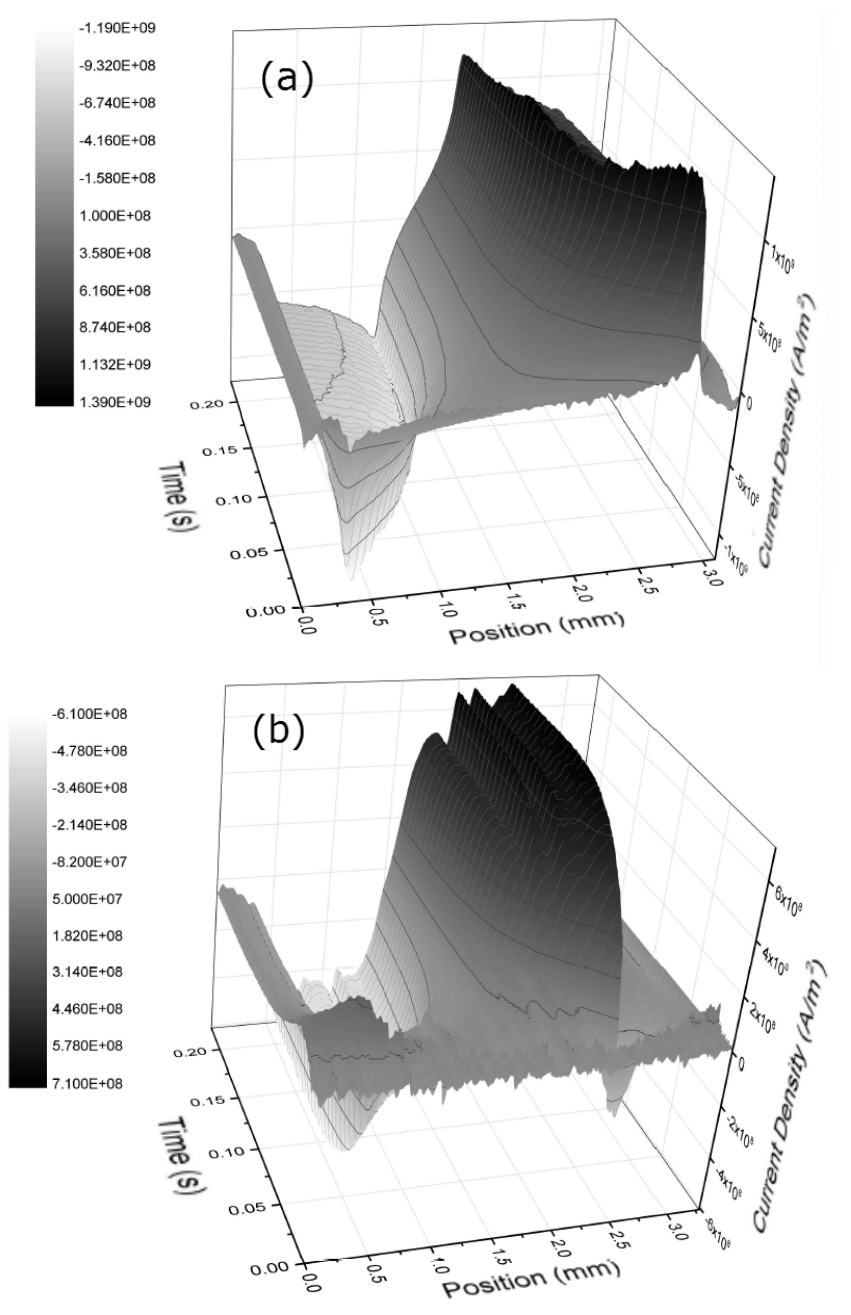

FIG. 6. 3D plots of time-evolution of current in (a) the highquality sample, and (b) the reduced-quality sample, as calculated frame-by-frame from magneto-optical video during flux penetration. Any vertical slice of these plots taken perpendicular to the time axis represents the one-dimensional current profile in the sample at a given point in time.

to $0.8 \mathrm{~mm}$, so the small current corresponding to this flux is indistinguishable from the background, and hence interpreted as zero). After the field has stabilised, the deviations become more drastic, with the peak in current not moving from the sample edge at all. The current distribution still spreads toward the sample centre as flux penetrates further inwards, but has a rounded shape with downward concavity, rather than a sharp peak at the flux front. Minimal currents are visualised in the non-penetrated region at all times for this sample. The current can still be interpreted as having a plateau in the penetrated region, but with a reversed gradient that is much steeper. After $100 \mathrm{~ms}$, as flux has penetrated almost to the sample centre, the current plateau becomes much less smooth. Perturbations in the plateau appear as ridges, which are non-symmetrically distributed about the centre, suggesting that these are caused by defects.
The final current distribution in the reduced-quality sample deviates strongly from both Bean- and Kim-like profiles, with the current plateau having a gradient sloping toward the sample centre and peak current at the edge. Perturbations in this plateau attributed to strong current-limiting defects are still very much apparent.

It should be noted that the maximum value for current calculated from MO images cannot be directly compared to the $J_{c}$ found by MPMS. This is partially due to differences in measurement techniques [34]. While the MOI technique is useful for determining the relative strength of current and local variations throughout the film, the magnitude of current calculated cannot be so easily compared from one sample to another. Errors arise in the calculation of absolute current in the sample due to quantification and variations in light intensity.

For both samples, the magnitude of current in all parts of the film decreases slightly in the longer time-scale up to $500 \mathrm{~ms}$ (not shown) due to relaxation processes, but the distribution retains approximately the same shape. It is only at this later time that non-zero currents occur close to the centre of the reduced-quality sample.

\section{CONCLUSION}

We have employed magneto-optical imaging in a dynamic and quantitative manner for two YBCO films with very different current-carrying abilities.

Vortex velocity is expected to increase with a reduced critical current [40], however the inward speed of vortices at the flux front appeared to decrease in the damaged sample. Upon further investigation, it was shown that initial flux penetration in the damaged film is probably in fact much faster. However, this initial penetration occurs so quickly that the time resolution of imaging used in this work was too low to resolve the initial fast flux penetration in this film. Therefore, future magneto-optical investigations will be performed with even faster video and utilise the apparatus to its full potential.

For a more complete understanding, we also present an analysis of current evolution in the films by Biot-Savart inversion, shown to be valid in our dynamic regime, at each time step. The final, quasi-equilibrium current profile in the high quality film reproduces the expected Kimmodel [13-16], whereas the damaged film does not exhibit either Kim-like or Bean profiles.

The suppression of the macroscopic critical current density in the reduced-quality sample as measured by MPMS is seen as a major influence on the dynamic evolution of current in the damaged sample. And conversely, dynamic MOI is shown to be useful for probing current evolution on a local scale in order to explain the dramatic reduction in overall critical current that was seen with MPMS.

Overall, we have presented an extension of the magneto-optical imaging technique to allow dynamic 
analysis of the field and current of superconducting samples using high-speed magneto-optical video.

\section{ACKNOWLEDGEMENT}

This work is supported by the Australian Research Council and the University of Wollongong.

[1] A. V. Pan, S. V. Pysarenko, S. X. Dou (2009) "Quantitative Description of Critical Current Density in YBCO Films and Multilayers" IEEE Trans Appl. Supercond. 19, 3391

[2] C. Jooss et al. (1998) "High-resolution magneto-optical imaging of critical currents in $\mathrm{YBa}_{2} \mathrm{Cu}_{3} \mathrm{O}_{7-\delta}$ thin films" Phys. C 299, 215

[3] A. Polyanskii, R. L. S. Emergo, J. Z. Wu, T. Aytug, D. K. Christen, G. K. Perkins, and D. Larbalestier (2005) "Magneto-optical imaging and electromagnetic study of $\mathrm{YBa}_{2} \mathrm{Cu}_{3} \mathrm{O}_{7}$ vicinal films of variable thickness", Phys. Rev. B 72, 174509.

[4] V. K. Vlasko-Vlasov, G. W. Crabtree, U. Welp, V. Nikitenko (1999) Magneto-Optical Studies of Magnetisation Processes in High- $T_{c}$ Superconductors, Physics and Materials Science of Vortex States, Flux Pinning and Dynamics, ed. R. Kossowsky, Springer Science \& Business Media, Turkey, pp. 205-208.

[5] A. V. Pan, S. X. Dou, H. K. Liu (2003) "Magnetic flux distribution in a superconducting core of Bi-2223 tape" Physica C 388-389, 405

[6] Q.-P. Ding, S. Mohan, Y. Tsuchiya, T. Taen, Y. Nakajima, T. Tamegai (2012) "Magneto-optical imaging and transport properties of FeSe superconducting tapes prepared by the diffusion method" Supercond. Sci. Technol. 25, 025003

[7] H. Liu, A. Polyanskii, W. M. Chen, Y. C. Guo, S. X. Dou (2001) "Magneto-optical images of Ag/Bi-2223 tapes processed by flat rolling, 'sandwich' rolling and pressing" IEEE Trans. Appl. Supercond. 11, 3764-3767

[8] D.C. van der Laan, H. J. N. van Eck, M. W. Davidson, B. ten Haken, H. H. J. ten Kate, J. Schwartz (2002) "Magneto-optical imaging study of the crack formation in superconducting tapes caused by applied strain" Physica C 372-376, 1020-1023

[9] M. Roussel, A. V. Pan, A. V. Bobyl, Y. Zhao, S. X. Dou, T. H. Johansen (2005) "Magnetic flux penetration in $\mathrm{MgB}_{2}$ thin films produced by pulsed laser deposition" Supercond. Sci. Technol. 18, 1391

[10] A. V. Pan, Y. Zhao, M. Ionescu, S.X. Dou, V. A. Komashko, V. S. Flis, V. M. Pan (2004) "Thermally activated depinning of individual vortices in $\mathrm{YBa}_{2} \mathrm{Cu}_{3} \mathrm{O}_{7}$ superconducting films" Physica C 407, 10

[11] A. V. Pan, S. X. Dou (2006) "Comparison of small-field behavior in $\mathrm{MgB} 2$, low- and high-temperature superconductors" Phys. Rev. B 73, 052506

[12] C. Jooss et al. (2002) "Magneto-optical studies of current distributions in high- $\mathrm{T}_{c}$ superconductors" Rep. Prog. Phys. 65, 651

[13] J. McDonald, J. R. Clem (1996) "Theory of flux penetration into thin films with field-dependent critical current"
Phys. Rev. B 53, 8643

[14] D. V. Shantsev, Y. M. Galperin, T. H. Johansen (1999) 'Thin superconducting disk with B-dependent $\mathrm{J}_{c}$ : Flux and current distributions" Phys. Rev. B 60, 13112

[15] Y. B. Kim, C. F. Hempstead, A. R. Strand (1962) "Critical Persistent Currents in Hard Superconductors", Phys. Rev. Lett. 9, 306

[16] Y. B. Kim, C. F. Hempstead, A. R. Strand (1963) "Magnetization and Critical Supercurrents" Phys. Rev. 129, 528

[17] F. Grilli, A. Lucarelli, G. Lüpke, T. Haugan, P. Barnes (2008) "Dynamic Study of Field and Current Distribution in Multifilamentary YBCO Thin Films" Proceedings of the COMSOL Conference Boston

[18] E. Farber, G. Deutscher, J. P. Contour, and E. Jerby (1998) "Penetration depth measurement in high quality $\mathrm{YBa}_{2} \mathrm{Cu}_{3} \mathrm{O}_{7-x}$ thin films" Eur. Phys. J. B 5, 159-162

[19] M. R. Koblischka, T. H. Johansen, H. Bratsberg, L. P., Y. Shen, P. Vase (1998) "Magneto-optical observation of dynamic relaxation in thin films" J. Phys. Cond. Mat. 9, 10909

[20] A. V. Bobyl, D. V. Shantsev, Y. M. Galperin, T. H. Johansen, M. Baziljevich, S. F. Karmanenko (2002) "Relaxation of transport current distribution in a $\mathrm{YBaCuO}$ strip studied by magneto-optical imaging" Supercond. Sci. Technol. 15, 82-89

[21] A. Lucarelli, G. Lüpke, T. J. Haugan, G. A. Levin, P. N. Barnes (2006) "Time-resolved magneto-optical imaging of $\mathrm{Y}_{1} \mathrm{Ba}_{2} \mathrm{Cu}_{3} \mathrm{O}_{7-\delta}$ thin films in high-frequency AC current regime" Supercond. Sci. Technol. 19, 667-670

[22] H. Song, M. W. Davidson, J. Schwartz (2009) “Dynamic magneto-optical imaging of transport current redistribution and normal zone propagation in $\mathrm{YBa}_{2} \mathrm{Cu}_{3} \mathrm{O}_{7-\delta}$ coated conductor" Supercond. Sci. Technol. 22, 062001

[23] H. Murakami, R. Kitamura, I. Kawayama, M. Tonouchi (2009) "Development of a high-speed and a high-sensitive laser scanning magneto-optical imaging system JPCS 150, 012029

[24] R. J. Wijngaarden, K. Heeck, M. Welling, R. Limburg, M. Pannetier, K. van Zetten, V. L. G. Roorda, A. R. Voorwinden (2001) "Fast imaging polarimeter for magneto-optical investigations Rev. Sci. Instrum. 72, 2661

[25] Y. Yeshurun, A. P.Malozemoff, and A. Shaulov (1996) "Magnetic relaxation in high-temperature superconductors" Rev.Mod. Phys. 68, 911

[26] F. S. Wells, A. V. Pan, X. R. Wang,S. A. Fedoseev, H. Hilgenkamp (2015) "Analysis of low-field isotropic vortex glass containing vortex groups in $\mathrm{YBa}_{2} \mathrm{Cu}_{3} \mathrm{O}_{7-x}$ thin films visualized by scanning SQUID microscopy", Sci. Rep. 5, 8677 
[27] F. S. Wells (2011) "Magneto-optical imaging and current profiling on superconductors", Honours Thesis, University of Wollongong

[28] T. Johansen et al. (1996) "Direct observation of the current distribution in thin superconducting strips using magneto-optic imaging." Phys. Rev. B 54, 16264

[29] R. J. Wijngaarden, K. Heeck, H.J.W. Spoelder, R. Surdeanu, R. Griessen (1998) "Fast determination of 2D current patterns in flat conductors from measurement of their magnetic field" Physica C 295, 177-185

[30] P. Paturi (1995) "Taking the horizontal fields intrinsically into account in magneto-optical microscopy" Rev. Sci. Instrum. 76, 093908

[31] I. A. Golovchanskiy, A. V. Pan, S. A. Fedoseev, M. Higgins (2014) "Significant tunability of thin film functionalities enabled bymanipulating magnetic and structural nano-domains" Appl. Surf. Sci. 311, 549.

[32] A. V. Pan, S. V. Pysarenko, D. Wexler, S. Rubanov, S. X. Dou (2007) "Multilayering and Ag-Doping for Properties and Performance Enhancement in $\mathrm{YBa}_{2} \mathrm{Cu}_{3} \mathrm{O}_{7}$ Films" IEEE Trans. Appl. Supercond. 17, 3585

[33] A. V. Pan, I. A. Golovchanskiy, S. A. Fedoseev (2013) "Critical current density: Measurements vs. reality" Europhys. Lett. 103, 17006
[34] I. A. Golovchanskiy, A. V. Pan, O. V. Shcherbakova, S. A. Fedoseev (2013) "Rectifying differences in transport, dynamic, and quasi-equilibrium measurements of critical current density" J. Appl. Phys. 114, 163910

[35] R. Wolfe, E. M. Gyorgy, R. A. Lieberman, V. J. Fratello, S. J. Licht, M. N. Deeter, G. W. Day (1992) "High frequency magnetic field sensors based on the Faraday effect in garnet thick films Appl. Phys. Lett. 60, 2048

[36] D. J. Griffiths (1998) Introduction to Electrodynamics (3rd ed.). Prentice Hall. pp. 222-224

[37] E. H. Brandt (1995) "Electric field in superconductors with rectangular cross-section" Phys. Rev. B 52, 15442

[38] C. Romero-Salazar, Ch. Jooss, O. A. Hernandez-Flores (2010) "Reconstruction of the electric field in type-II superconducting thin films in perpendicular geometry" Phys. Rev. B 81, 144506

[39] S. J. Di Bartolo, A. T. Dorsey (1996) "Velocity Selection for Propagating Fronts in Superconductors Phys. Rev. Lett. 77, 4442

[40] Th. Schuster, H. Kuhn, E. H. Brandt, S. Klaumünzer (1997) "Flux penetration into flat rectangular superconductors with anisotropic critical current" Phys. Rev. $B$ 56, 3413

[41] V. Pan, Y. Cherpak, V. Komashko, S. Pozigun, C. Tretiatchenko, A. Semenov, E. Pashitskii, A. V. Pan (2006) "Supercurrent transport in $\mathrm{YBa}_{2} \mathrm{Cu}_{3} \mathrm{O}_{7-\delta}$ epitaxial thin films in a dc magnetic field" Phys. Rev B 73, 054508 